\title{
Differentiation in Normed Spaces ${ }^{1}$
}

\author{
Noboru Endou \\ Gifu National College of Technology \\ Japan
}

\author{
Yasunari Shidama \\ Shinshu University \\ Nagano, Japan
}

Summary. In this article we formalized the Fréchet differentiation. It is defined as a generalization of the differentiation of a real-valued function of a single real variable to more general functions whose domain and range are subsets of normed spaces [14].

MSC: 58C20 46G05 03B35

Keywords: formalization of Fréchet derivative; Fréchet differentiability

MML identifier: NDIFF_6, version: 8.1.02 5.17.1179

The notation and terminology used in this paper have been introduced in the following articles: [5], [1], 4], 10], 6], [7], [16], 15], [11, [12], [13], [3], 8], [19], [20], [17], 18], 21], and [9].

Let us consider non empty sets $D, E, F$. Now we state the propositions:

(1) There exists a function $I$ from $\left(F^{E}\right)^{D}$ into $F^{D \times E}$ such that

(i) $I$ is bijective, and

(ii) for every function $f$ from $D$ into $F^{E}$ and for every elements $d, e$ such that $d \in D$ and $e \in E$ holds $I(f)(d, e)=f(d)(e)$.

(2) There exists a function $I$ from $\left(F^{E}\right)^{D}$ into $F^{E \times D}$ such that

(i) $I$ is bijective, and

(ii) for every function $f$ from $D$ into $F^{E}$ and for every elements $e, d$ such that $e \in E$ and $d \in D$ holds $I(f)(e, d)=f(d)(e)$.

Now we state the propositions:

(3) Let us consider non-empty non empty finite sequences $D, E$ and a non empty set $F$. Then there exists a function $L$ from $\left(F \prod E\right) \prod D$ into $F \prod\left(E^{\wedge} D\right)$ such that

(i) $L$ is bijective, and

\footnotetext{
${ }^{1}$ This work was supported by JSPS KAKENHI 23500029 and 22300285. 
(ii) for every function $f$ from $\prod D$ into $F \prod^{E}$ and for every finite sequen-

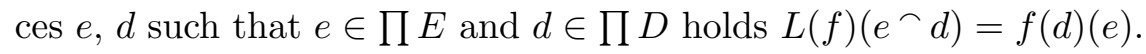

The theorem is a consequence of (2). Proof: Consider $I$ being a function from $\left(F \prod^{E}\right) \prod^{D}$ into $F \prod E \times \prod^{D}$ such that $I$ is bijective and for every function $f$ from $\prod D$ into $F \prod^{E}$ and for every elements $e, d$ such that $e \in \prod E$ and $d \in \prod D$ holds $I(f)(e, d)=f(d)(e)$. Consider $J$ being a function from $\prod E \times \prod D$ into $\prod\left(E^{\frown} D\right)$ such that $J$ is one-to-one and onto and for every finite sequences $x, y$ such that $x \in \prod E$ and $y \in \prod D$ holds $J(x, y)=x^{\frown} y$. Reconsider $K=J^{-1}$ as a function from $\Pi\left(E^{\frown} D\right)$ into $\prod E \times \prod D$. Define $\mathcal{G}$ (element) $=I\left(\$_{1}\right) \cdot K$. For every element $x$ such that $x \in\left(F \prod^{E}\right) \prod^{D}$ holds $\mathcal{G}(x) \in F \prod^{\left(E^{\frown} D\right)}$ by [7, (5), (8), (128)]. Consider $L$ being a function from $\left(F \prod E\right) \prod^{D}$ into $F \prod^{\left(E^{-} D\right)}$ such that for every element $e$ such that $e \in\left(F \prod^{E}\right) \prod^{D}$ holds $L(e)=\mathcal{G}(e)$ from [7, Sch. 2]. For every function $f$ from $\prod D$ into $F \prod E$ and for every finite sequences $e, d$ such that $e \in \prod E$ and $d \in \prod D$ holds $L(f)\left(e^{\frown} d\right)=f(d)(e)$ by [9, (87)], [7, (26), (8), (5)].

(4) Let us consider non empty sets $X, Y$. Then there exists a function $I$ from $X \times Y$ into $X \times \prod\langle Y\rangle$ such that

(i) $I$ is bijective, and

(ii) for every elements $x, y$ such that $x \in X$ and $y \in Y$ holds $I(x, y)=\langle x$, $\langle y\rangle\rangle$.

Proof: Consider $J$ being a function from $Y$ into $\prod\langle Y\rangle$ such that $J$ is oneto-one and onto and for every element $y$ such that $y \in Y$ holds $J(y)=\langle y\rangle$. Define $\mathcal{P}$ [element, element, element] $\equiv \$_{3}=\left\langle \$_{1},\left\langle \$_{2}\right\rangle\right\rangle$. For every elements $x, y$ such that $x \in X$ and $y \in Y$ there exists an element $z$ such that $z \in X \times \Pi\langle Y\rangle$ and $\mathcal{P}[x, y, z]$ by [7, (5)], [9, (87)]. Consider $I$ being a function from $X \times Y$ into $X \times \prod\langle Y\rangle$ such that for every elements $x, y$ such that $x \in X$ and $y \in Y$ holds $\mathcal{P}[x, y, I(x, y)]$ from [5, Sch. 1].

(5) Let us consider a non-empty non empty finite sequence $X$ and a non empty set $Y$. Then there exists a function $K$ from $\prod X \times Y$ into $\prod(X \frown\langle Y\rangle)$ such that

(i) $K$ is bijective, and

(ii) for every finite sequence $x$ and for every element $y$ such that $x \in \prod X$ and $y \in Y$ holds $K(x, y)=x^{\frown}\langle y\rangle$.

The theorem is a consequence of (4). Proof: Consider $I$ being a function from $\prod X \times Y$ into $\prod X \times \prod\langle Y\rangle$ such that $I$ is bijective and for every element $x$ and for every element $y$ such that $x \in \prod X$ and $y \in Y$ holds $I(x, y)=\langle x,\langle y\rangle\rangle$. Consider $J$ being a function from $\prod X \times \prod\langle Y\rangle$ into $\Pi(X \frown\langle Y\rangle)$ such that $J$ is one-to-one and onto and for every finite sequences $x, y$ such that $x \in \prod X$ and $y \in \prod\langle Y\rangle$ holds $J(x, y)=x^{\frown} y$. Set 
$K=J \cdot I$. For every finite sequence $x$ and for every element $y$ such that $x \in \prod X$ and $y \in Y$ holds $K(x, y)=x^{\frown}\langle y\rangle$ by [9, (87)], [7, (5), (15)].

(6) Let us consider a non empty set $D$, a non-empty non empty finite sequence $E$, and a non empty set $F$. Then there exists a function $L$ from $\left(F \prod^{E}\right)^{D}$ into $F \prod^{\left(E^{-}\langle D\rangle\right)}$ such that

(i) $L$ is bijective, and

(ii) for every function $f$ from $D$ into $F \prod^{E}$ and for every finite sequence $e$ and for every element $d$ such that $e \in \prod E$ and $d \in D$ holds $L(f)\left(e^{\frown}\langle d\rangle\right)=f(d)(e)$.

The theorem is a consequence of (2) and (5). Proof: Consider $I$ being a function from $\left(F \prod^{E}\right)^{D}$ into $F \prod E \times D$ such that $I$ is bijective and for every function $f$ from $D$ into $F \prod^{E}$ and for every elements $e, d$ such that $e \in \prod E$ and $d \in D$ holds $I(f)(e, d)=f(d)(e)$. Consider $J$ being a function from $\prod E \times D$ into $\prod\left(E^{\frown}\langle D\rangle\right)$ such that $J$ is bijective and for every finite sequence $x$ and for every element $y$ such that $x \in \prod E$ and $y \in D$ holds $J(x, y)=x^{\frown}\langle y\rangle$. Reconsider $K=J^{-1}$ as a function from $\prod\left(E^{\frown}\langle D\rangle\right)$ into $\prod E \times D$. Define $\mathcal{G}($ element $)=I\left(\$_{1}\right) \cdot K$. For every element $x$ such that $x \in\left(F \prod E\right)^{D}$ holds $\mathcal{G}(x) \in F \prod^{\left(E^{\frown}\langle D\rangle\right)}$ by [7, (5), (8), (128)]. Consider $L$ being a function from $\left(F \prod^{E}\right)^{D}$ into $F \prod^{\left(E^{\wedge}\langle D\rangle\right)}$ such that for every element $e$ such that $e \in\left(F \prod^{E}\right)^{D}$ holds $L(e)=\mathcal{G}(e)$ from [7, Sch. 2]. For every function $f$ from $D$ into $F \prod^{E}$ and for every finite sequence $e$ and for every element $d$ such that $e \in \prod E$ and $d \in D$ holds $L(f)\left(e^{\frown}\langle d\rangle\right)=f(d)(e)$ by [7. (5), (26), (8)].

In this paper $S, T$ denote real normed spaces, $f, f_{1}, f_{2}$ denote partial functions from $S$ to $T, Z$ denotes a subset of $S$, and $i, n$ denote natural numbers.

Let $S$ be a set. Assume $S$ is a real normed space. The functor $\operatorname{NormSp}_{\mathbb{R}}(S)$ yielding a real normed space is defined by the term

(Def. 1) $S$.

Let $S, T$ be real normed spaces. The functor $\operatorname{diff}_{\mathrm{SP}}(S, T)$ yielding a function is defined by

(Def. 2) (i) $\operatorname{dom} i t=\mathbb{N}$, and

(ii) $i t(0)=T$, and

(iii) for every natural number $i, i t(i+1)=$ the real norm space of bounded linear operators from $S$ into $\operatorname{NormSp}_{\mathbb{R}}(i t(i))$.

Now we state the proposition:

(i) $\left(\operatorname{diff}_{\mathrm{SP}}(S, T)\right)(0)=T$, and

(ii) $\left(\operatorname{diff}_{\mathrm{SP}}(S, T)\right)(1)=$ the real norm space of bounded linear operators from $S$ into $T$, and 
(iii) $\left(\operatorname{diff}_{\mathrm{SP}}(S, T)\right)(2)=$ the real norm space of bounded linear operators from $S$ into the real norm space of bounded linear operators from $S$ into $T$.

Let us consider a natural number $i$. Now we state the propositions:

(8) $\quad\left(\operatorname{diff}_{\mathrm{SP}}(S, T)\right)(i)$ is a real normed space.

(9) There exists a real normed space $H$ such that

(i) $H=\left(\operatorname{diff}_{\mathrm{SP}}(S, T)\right)(i)$, and

(ii) $\left(\operatorname{diff}_{\mathrm{SP}}(S, T)\right)(i+1)=$ the real norm space of bounded linear operators from $S$ into $H$.

Let $S, T$ be real normed spaces and $i$ be a natural number. The functor $\operatorname{diff}_{\mathrm{SP}}\left(S^{i}, T\right)$ yielding a real normed space is defined by the term

(Def. 3) $\left(\operatorname{diff}_{\mathrm{SP}}(S, T)\right)(i)$.

Now we state the proposition:

(10) Let us consider a natural number $i$. Then $\operatorname{diff}_{\mathrm{SP}}\left(S^{(i+1)}, T\right)=$ the real norm space of bounded linear operators from $S$ into $\operatorname{diff}_{\mathrm{SP}}\left(S^{i}, T\right)$. The theorem is a consequence of (9).

Let $S, T$ be real normed spaces and $f$ be a set. Assume $f$ is a partial function from $S$ to $T$. The functor $\operatorname{PartFuncs}(f, S, T)$ yielding a partial function from $S$ to $T$ is defined by the term

(Def. 4) $f$.

Let $f$ be a partial function from $S$ to $T$ and $Z$ be a subset of $S$. The functor $f^{\prime}(Z)$ yielding a function is defined by

(Def. 5) (i) $\operatorname{dom} i t=\mathbb{N}$, and

(ii) it $(0)=f\lceil Z$, and

(iii) for every natural number $i, i t(i+1)=$ $\left(\operatorname{PartFuncs}\left(i t(i), S, \operatorname{diff}_{\mathrm{SP}}\left(S^{i}, T\right)\right)\right)^{\prime}{ }_{Z}$.

Now we state the propositions:

(i) $f^{\prime}(Z)(0)=f\lceil Z$, and

(ii) $f^{\prime}(Z)(1)=\left(f\lceil Z)_{\uparrow Z}^{\prime}\right.$, and

(iii) $f^{\prime}(Z)(2)=\left(\left(f\lceil Z)^{\prime}{ }_{\mid Z}\right)_{\lceil Z}^{\prime}\right.$.

The theorem is a consequence of $(7)$.

(12) Let us consider a natural number $i$. Then $f^{\prime}(Z)(i)$ is a partial function from $S$ to $\operatorname{diff}_{\mathrm{SP}}\left(S^{i}, T\right)$. The theorem is a consequence of (7). Proof: Define $\mathcal{P}$ [natural number] $\equiv f^{\prime}(Z)\left(\$_{1}\right)$ is a partial function from $S$ to $\operatorname{diff}_{\mathrm{SP}}\left(S^{\$_{1}}, T\right)$. For every natural number $n, \mathcal{P}[n]$ from [2, Sch. 2].

Let $S, T$ be real normed spaces, $f$ be a partial function from $S$ to $T, Z$ be a subset of $S$, and $i$ be a natural number. The functor $\operatorname{diff}_{Z}(f, i)$ yielding a partial function from $S$ to $\operatorname{diff}_{\mathrm{SP}}\left(S^{i}, T\right)$ is defined by the term 
(Def. 6) $f^{\prime}(Z)(i)$.

Now we state the proposition:

(13) $\operatorname{diff}_{Z}(f, i+1)=\operatorname{diff}_{Z}(f, i)^{\prime}{ }_{Z}$. The theorem is a consequence of (12) and (8).

Let $S, T$ be real normed spaces, $f$ be a partial function from $S$ to $T, Z$ be a subset of $S$, and $n$ be a natural number. We say that $f$ is differentiable $n$ times on $Z$ if and only if

(Def. 7) (i) $Z \subseteq \operatorname{dom} f$, and

(ii) for every natural number $i$ such that $i \leqslant n-1$ holds

$\operatorname{PartFuncs}\left(f^{\prime}(Z)(i), S, \operatorname{diff}_{\mathrm{SP}}\left(S^{i}, T\right)\right)$ is differentiable on $Z$.

Now we state the propositions:

(14) $f$ is differentiable $n$ times on $Z$ if and only if $Z \subseteq \operatorname{dom} f$ and for every natural number $i$ such that $i \leqslant n-1$ holds $\operatorname{diff}_{Z}(f, i)$ is differentiable on $Z$.

(15) $f$ is differentiable 1 times on $Z$ if and only if $Z \subseteq \operatorname{dom} f$ and $f\lceil Z$ is differentiable on $Z$. The theorem is a consequence of (14) and (7). ProOF: For every natural number $i$ such that $i \leqslant 1-1$ holds $\operatorname{diff}_{Z}(f, i)$ is differentiable on $Z$.

(16) $f$ is differentiable 2 times on $Z$ if and only if $Z \subseteq \operatorname{dom} f$ and $f\lceil Z$ is differentiable on $Z$ and $\left(f\lceil Z)^{\prime}{ }_{\mid Z}\right.$ is differentiable on $Z$. The theorem is a consequence of (14), (7), and (11). Proof: For every natural number $i$ such that $i \leqslant 2-1$ holds $\operatorname{diff}_{Z}(f, i)$ is differentiable on $Z$ by [2, (14)].

(17) Let us consider real normed spaces $S, T$, a partial function $f$ from $S$ to $T$, a subset $Z$ of $S$, and a natural number $n$. Suppose $f$ is differentiable $n$ times on $Z$. Let us consider a natural number $m$. If $m \leqslant n$, then $f$ is differentiable $m$ times on $Z$.

(18) Let us consider a natural number $n$ and a partial function $f$ from $S$ to $T$. If $1 \leqslant n$ and $f$ is differentiable $n$ times on $Z$, then $Z$ is open. The theorem is a consequence of (17) and (15).

(19) Let us consider a natural number $n$ and a partial function $f$ from $S$ to T. Suppose

(i) $1 \leqslant n$, and

(ii) $f$ is differentiable $n$ times on $Z$.

Let us consider a natural number $i$. Suppose $i \leqslant n$. Then

(iii) $\left(\operatorname{diff}_{\mathrm{SP}}(S, T)\right)(i)$ is a real normed space, and

(iv) $f^{\prime}(Z)(i)$ is a partial function from $S$ to $\operatorname{diff}_{\mathrm{SP}}\left(S^{i}, T\right)$, and

(v) $\operatorname{dom} \operatorname{diff}_{Z}(f, i)=Z$.

The theorem is a consequence of (13) and (14). 
(20) Let us consider a natural number $n$ and partial functions $f, g$ from $S$ to $T$. Suppose

(i) $1 \leqslant n$, and

(ii) $f$ is differentiable $n$ times on $Z$, and

(iii) $g$ is differentiable $n$ times on $Z$.

Let us consider a natural number $i$. Suppose $i \leqslant n$. Then $\operatorname{diff}_{Z}(f+g, i)=$ $\operatorname{diff}_{Z}(f, i)+\operatorname{diff}_{Z}(g, i)$. The theorem is a consequence of (18), (14), (19), (13), and (10). Proof: Define $\mathcal{P}$ [natural number] $\equiv$ if $\$_{1} \leqslant n$, then $\operatorname{diff}_{Z}\left(f+g, \$_{1}\right)=\operatorname{diff}_{Z}\left(f, \$_{1}\right)+\operatorname{diff}_{Z}\left(g, \$_{1}\right)$. $\mathcal{P}[0]$ by [21, (27)]. For every natural number $i$ such that $\mathcal{P}[i]$ holds $\mathcal{P}[i+1]$ by [2, (11)], [11, (39)], [8. (5)]. For every natural number $n, \mathcal{P}[n]$ from [2, Sch. 2].

(21) Let us consider a natural number $n$ and partial functions $f, g$ from $S$ to T. Suppose

(i) $1 \leqslant n$, and

(ii) $f$ is differentiable $n$ times on $Z$, and

(iii) $g$ is differentiable $n$ times on $Z$.

Then $f+g$ is differentiable $n$ times on $Z$. The theorem is a consequence of (18), (14), (19), and (20). Proof: For every natural number $i$ such that $i \leqslant n-1$ holds $\operatorname{diff}_{Z}(f+g, i)$ is differentiable on $Z$ by [11, (39)].

(22) Let us consider a natural number $n$ and partial functions $f, g$ from $S$ to T. Suppose

(i) $1 \leqslant n$, and

(ii) $f$ is differentiable $n$ times on $Z$, and

(iii) $g$ is differentiable $n$ times on $Z$.

Let us consider a natural number $i$. Suppose $i \leqslant n$. Then $\operatorname{diff}_{Z}(f-g, i)=$ $\operatorname{diff}_{Z}(f, i)-\operatorname{diff}_{Z}(g, i)$. The theorem is a consequence of (18), (14), (19), (13), and (10). Proof: Define $\mathcal{P}$ [natural number] $\equiv$ if $\$_{1} \leqslant n$, then $\operatorname{diff}_{Z}\left(f-g, \$_{1}\right)=\operatorname{diff}_{Z}\left(f, \$_{1}\right)-\operatorname{diff}_{Z}\left(g, \$_{1}\right) . \mathcal{P}[0]$ by [21, (30)]. For every natural number $i$ such that $\mathcal{P}[i]$ holds $\mathcal{P}[i+1]$ by [2, (11)], [11, (40)], [8, (5)]. For every natural number $n, \mathcal{P}[n]$ from [2, Sch. 2].

(23) Let us consider a natural number $n$ and partial functions $f, g$ from $S$ to T. Suppose

(i) $1 \leqslant n$, and

(ii) $f$ is differentiable $n$ times on $Z$, and

(iii) $g$ is differentiable $n$ times on $Z$.

Then $f-g$ is differentiable $n$ times on $Z$. The theorem is a consequence of (18), (14), (19), and (22). Proof: For every natural number $i$ such that $i \leqslant n-1$ holds $\operatorname{diff}_{Z}(f-g, i)$ is differentiable on $Z$ by [11, (40)]. 
(24) Let us consider a natural number $n$, a real number $r$, and a partial function $f$ from $S$ to $T$. Suppose

(i) $1 \leqslant n$, and

(ii) $f$ is differentiable $n$ times on $Z$.

Let us consider a natural number $i$. If $i \leqslant n$, then $\operatorname{diff}_{Z}(r \cdot f, i)=r$. $\operatorname{diff}_{Z}(f, i)$. The theorem is a consequence of (18), (14), (19), (10), and (13). Proof: Define $\mathcal{P}$ [natural number] $\equiv$ if $\$_{1} \leqslant n$, then $\operatorname{diff}_{Z}\left(r \cdot f, \$_{1}\right)=$ $r \cdot \operatorname{diff}_{Z}\left(f, \$_{1}\right) \cdot \mathcal{P}[0]$ by [21, (31)]. For every natural number $i$ such that $\mathcal{P}[i]$ holds $\mathcal{P}[i+1]$ by [2, (11)], [11, (41)]. For every natural number $n$, $\mathcal{P}[n]$ from [2, Sch. 2].

(25) Let us consider a natural number $n$, a real number $r$, and a partial function $f$ from $S$ to $T$. Suppose

(i) $1 \leqslant n$, and

(ii) $f$ is differentiable $n$ times on $Z$.

Then $r \cdot f$ is differentiable $n$ times on $Z$. The theorem is a consequence of (18), (14), (24), and (19). Proof: For every natural number $i$ such that $i \leqslant n-1$ holds $\operatorname{diff}_{Z}(r \cdot f, i)$ is differentiable on $Z$ by [11, (41)].

(26) Let us consider a natural number $n$ and a partial function $f$ from $S$ to T. Suppose

(i) $1 \leqslant n$, and

(ii) $f$ is differentiable $n$ times on $Z$.

Let us consider a natural number $i$. Suppose $i \leqslant n$. Then $\operatorname{diff}_{Z}(-f, i)=$ $-\operatorname{diff}_{Z}(f, i)$. The theorem is a consequence of (24).

(27) Let us consider a natural number $n$ and a partial function $f$ from $S$ to T. Suppose

(i) $1 \leqslant n$, and

(ii) $f$ is differentiable $n$ times on $Z$.

Then $-f$ is differentiable $n$ times on $Z$. The theorem is a consequence of (25).

\section{REFERENCES}

[1] Grzegorz Bancerek. König's theorem Formalized Mathematics, 1(3):589-593, 1990.

[2] Grzegorz Bancerek. The fundamental properties of natural numbers. Formalized Mathematics, 1(1):41-46, 1990.

[3] Grzegorz Bancerek. The ordinal numbers Formalized Mathematics, 1(1):91-96, 1990.

[4] Grzegorz Bancerek and Krzysztof Hryniewiecki. Segments of natural numbers and finite sequences Formalized Mathematics, 1(1):107-114, 1990.

[5] Czesław Byliński. Binary operations. Formalized Mathematics, 1(1):175-180, 1990.

[6] Czesław Byliński. Functions and their basic properties Formalized Mathematics, 1(1): $55-65,1990$. 
[7] Czesław Byliński. Functions from a set to a set Formalized Mathematics, 1(1):153-164, 1990.

[8] Czesław Byliński. Partial functions Formalized Mathematics, 1(2):357-367, 1990.

[9] Czesław Byliński. Some basic properties of sets Formalized Mathematics, 1(1):47-53, 1990.

[10] Agata Darmochwał. Finite sets. Formalized Mathematics, 1(1):165-167, 1990.

[11] Hiroshi Imura, Morishige Kimura, and Yasunari Shidama. The differentiable functions on normed linear spaces Formalized Mathematics, 12(3):321-327, 2004.

[12] Takaya Nishiyama, Keiji Ohkubo, and Yasunari Shidama. The continuous functions on normed linear spaces Formalized Mathematics, 12(3):269-275, 2004.

[13] Jan Popiołek. Real normed space Formalized Mathematics, 2(1):111-115, 1991.

[14] Laurent Schwartz. Cours d'analyse. Hermann, 1981.

[15] Yasunari Shidama. Banach space of bounded linear operators Formalized Mathematics, 12(1):39-48, 2004.

[16] Michał J. Trybulec. Integers Formalized Mathematics, 1(3):501-505, 1990.

[17] Wojciech A. Trybulec. Vectors in real linear space. Formalized Mathematics, 1(2):291-296, 1990.

[18] Zinaida Trybulec. Properties of subsets. Formalized Mathematics, 1(1):67-71, 1990.

[19] Edmund Woronowicz. Relations and their basic properties. Formalized Mathematics, 1 (1):73-83, 1990.

[20] Edmund Woronowicz. Relations defined on sets. Formalized Mathematics, 1(1):181-186, 1990.

[21] Hiroshi Yamazaki and Yasunari Shidama. Algebra of vector functions. Formalized Mathematics, 3(2):171-175, 1992.

Received May 19, 2013 